\title{
Screening for thyroid dysfunction: prevention of overdiagnosis and overtreatment
}

\author{
Omar M. El Kawkgi MB BCh BAO, Juan P. Brito MD MSc
}

n Cite as: CMAJ 2019 November 18;191:E1260-1. doi: 10.1503/cmaj.191437

See related article at www.cmaj.ca/lookup/doi/10.1503/cmaj.190395

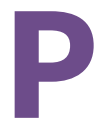

atient-centred care requires clinicians and patients to cocreate a care plan that responds to the situation of each patient in a manner consistent with the patient's values, preferences and priorities, based on the best research evidence. Care that responds poorly to a person's situation, is not evidence based, or fails to align with the patient's priorities and preferences is unlikely to be helpful and could be harmful. ${ }^{1}$ One common practice that may not represent patient-centred care is screening for thyroid dysfunction. In a linked guideline, the Canadian Task Force on Preventive Health Care strongly recommends against screening for thyroid dysfunction. ${ }^{2}$ This recommendation was made after assessing the limitations of the screening test (thyroid-stimulating hormone $[\mathrm{TSH}]$ ) and a careful evaluation of the relative benefits, harms, costs and inconveniences of treating ( $v$. not treating) asymptomatic people with abnormal TSH levels. The recommendation represents a change in thinking about screening for thyroid dysfunction and contradicts other medical society recommendations that favour screening, particularly among older people. ${ }^{3}$

In the face of a low pre-test probability, the likelihood of thyroid disease in asymptomatic people with 1 abnormal TSH test result is very low. In a study following outpatients without thyroid disease over 5 years, $62 \%$ of TSH values normalized on subsequent testing without any intervention, ${ }^{4}$ which could be explained in part by variations in TSH secretion. Furthermore, TSH values are also affected by drugs, autoimmune diseases ${ }^{5}$ and problems with interference in the analytical assay, ${ }^{6}$ which can lead to false positives that result in misclassification of people with no clinical evidence of thyroid dysfunction.

Another factor that supports a recommendation against screening for thyroid dysfunction in those who are asymptomatic is the potential harm of finding asymptomatic people with thyroid dysfunction. A recent review noted that as many as $10 \%$ of people have elevated serum TSH levels with normal circulating thyroid hormone levels. ${ }^{7}$ Studies of treatment with levothyroxine (to normalize TSH levels) have not shown significant benefit in this population, ${ }^{8}$ suggesting that high TSH with normal thyroid hormone, often referred to as subclinical hypothyroidism, may not represent thyroid dysfunction.

\section{KEY POINTS}

- New Canadian clinical guidance recommends not screening for thyroid dysfunction in people who are asymptomatic - an approach that supports patient-centred care.

- Liberal screening by testing thyroid stimulating hormone levels is a common practice that likely uncovers subclinical disease for which treatment has not been shown to improve outcomes and which may lead to overdiagnosis- and overtreatment-related harms.

- The recommendation against screening is based on low-quality evidence and invites additional research on the applicability of the recommendation for populations not represented in available studies.

However, finding and treating people with subclinical hypothyroidism may have led to an observed rise in the number of prescriptions for levothyroxine in the last decade. In the United States, the number of levothyroxine prescriptions increased from 97 million in 2007 to 120 million in 2014, making levothyroxine the most prescribed medication in the US. ${ }^{9}$ Despite lack of benefit of this common practice, people taking levothyroxine for subclinical hypothyroidism will likely be burdened by lifestyle adaptations. For example, the drug cannot be taken at the same time as food or multivitamins, and patients must attend frequent clinic appointments for monitoring, with attendant out-ofpocket and opportunity costs (e.g., taking time off work). Alongside these personal tolls, health care utilization and expenditures are increased. ${ }^{10}$ As the threshold for treating mild subclinical disease with levothyroxine lowers, ${ }^{11}$ the likelihood of iatrogenic medical complications related to treatment also increases (e.g., the risk of dysrhythmias and fractures is increased in patients treated with levothyroxine to a suppressed TSH at a hazard ratio of 1.60 [95\% confidence interval (CI) 1.10 to 2.33 ] and 2.02, [95\% $\mathrm{Cl} 1.55$ to 2.62 ], respectively, compared with those treated on levothyroxine with normal TSH). ${ }^{12}$

Although exact population-level rates of screening for thyroid dysfunction are unknown, estimates suggest that it is a common practice. One primary care study from Toronto found that $71 \%$ of 135243 patients (aged $\geq 20 \mathrm{yr}$ ) without known 
thyroid disease and not on thyroid medication had their levels of TSH tested at least once over a 2-year period. ${ }^{13}$ In the United Kingdom, an estimated $18 \%$ to $25 \%$ of the adult population receives routine thyroid function testing each year. ${ }^{11}$ Thus, by issuing a strong recommendation against population screening for thyroid dysfunction, the task force may help physicians to stop uncovering a large reservoir of people with mild thyroid dysfunction who are unlikely to benefit from identification or treatment, thereby preventing the overdiagnosis and overtreatment of otherwise healthy people.

The linked guideline's recommendations are discordant with those of some other guidelines. A joint recommendation by the American Thyroid Association and American Association of Clinical Endocrinologists suggests that screening for hypothyroidism be considered in people older than 60 years, which translates into testing about 60 million people in the US. ${ }^{3}$ This guideline placed a high value on the potential benefit of finding people with overt hypothyroidism and improving their quality of life with subsequent treatment. However, the guideline was released in 2012 and could not consider clinical evidence published over the last 7 years, which has shown lack of benefit of levothyroxine treatment in people with subclinical hypothyroidism. However, the US Preventive Services Task Force issued an updated guideline in 2015 recommending against screening for thyroid dysfunction based on insufficient evidence. ${ }^{14}$

The Canadian task force recommendation does not apply to people who present with symptoms associated with thyroid dysfunction that are common and nonspecific. As many as $25 \%$ of people with normal thyroid hormone levels report 1 or 2 nonspecific symptoms that may be related to thyroid dysfunction (e.g., dry skin, hair loss, constipation, myalgia, fatigue, menstrual irregularities, low energy and weight gain), of whom only $10 \%$ have thyroid dysfunction. ${ }^{15}$ For these people, experts informally agree that case finding with TSH testing is appropriate.

The recommendation against screening from the Canadian task force is the latest addition to recommendations for other clinical practices highlighting the potential harms of screening for asymptomatic people who may not always benefit from finding or treating conditions early. Yet, given that this recommendation against thyroid dysfunction screening is based on low- quality evidence, it invites researchers to conduct trials to understand its applicability on populations not represented in available studies - for instance, trials on symptomatic or asymptomatic young people with subclinical hypothyroidism.

\section{References}

1. Kerr EA, Hayward RA. Patient- centered performance management: enhancing value for patients and health care systems. JAMA 2013;310:137-8.

2. Birtwhistle R, Morissette K, Dickinson JA, et al.; Canadian Task Force on Preventive Health Care. Recommendation on screening adults for asymptomatic thyroid dysfunction in primary care. CMAJ 2019;191:1275-80.

3. Garber JR, Cobin RH, Gharib H, et al.; American Association of Clinical Endocrinologists and American Thyroid Association Taskforce on Hypothyroidism in Adults. Clinical practice guidelines for hypothyroidism in adults: cosponsored by the American Association of Clinical Endocrinologists and the American Thyroid Association. Endocr Pract 2012;18:988-1028.

4. Meyerovitch J, Rotman-Pikielny P, Sherf M, et al. Serum thyrotropin measurements in the community: five-year follow-up in a large network of primary care physicians. Arch Intern Med 2007;167:1533-8.

5. Keffer JH. Preanalytical considerations in testing thyroid function. Clin Chem 1996;42:125-34.

6. Ismail AA, Walker PL, Barth JH, et al. Wrong biochemistry results: two case reports and observational study in 5310 patients on potentially misleading thyroid-stimulating hormone and gonadotropin immunoassay results. Clin Chem 2002;48:2023-9.

7. Biondi B, Cappola AR, Cooper DS. Subclinical hypothyroidism: a review. JAMA 2019;322:153-60.

8. Feller M, Snel M, Moutzouri E, et al. Association of thyroid hormone therapy with quality of life and thyroid-related symptoms in patients with subclinical hypothyroidism: a systematic review and meta-analysis. JAMA 2018;320:1349-59.

9. Rodriguez-Gutierrez R, Maraka S, Ospina NS, et al. Levothyroxine overuse: time for an about face? Lancet Diabetes Endocrinol 2017;5:246-8.

10. Chu JW, Crapo LM. The treatment of subclinical hypothyroidism is seldom necessary. J Clin Endocrinol Metab 2001;86:4591-9.

11. Taylor PN, Iqbal A, Minassian C, et al. Falling threshold for treatment of borderline elevated thyrotropin levels - balancing benefits and risks: evidence from a large community-based study. JAMA Intern Med 2014;174:32-9.

12. Flynn RW, Bonellie SR, Jung RT, et al. Serum thyroid-stimulating hormone concentration and morbidity from cardiovascular disease and fractures in patients on long-term thyroxine therapy. J Clin Endocrinol Metab 2010;95:186-93.

13. Greiver M, Mclsaac M, DelGiudice L, et al., editors. Patterns of thyroid screening and case detection in patients without thyroid disease in Canadian primary care [poster]. North American Primary Care Research Group - 2017 PracticeBased Research Network (PBRN) Conference; 2017 July 22-23 2017; Bethesda (MD).

14. LeFevre ML; U.S. Preventive Services Task Force. Screening for thyroid dysfunction: U.S. Preventive Services Task Force recommendation statement. Ann Intern Med 2015;162:641-50.

15. Canaris GJ, Manowitz NR, Mayor G, et al. The Colorado thyroid disease prevalence study. Arch Intern Med 2000;160:526-34.
Competing interests: Juan Brito reports that he is a member of the American Thyroid Association Guidelines and Statements Committee. No other competing interests were declared.

This article was solicited and has not been peer reviewed.

Affiliation: Knowledge and Evaluation Research Unit, Division of Endocrinology, Diabetes, Metabolism, and Nutrition, Mayo Clinic, Rochester, Minn.
Contributors: Both authors contributed to the conception and design of the work, drafted the manuscript, revised it critically for important intellectual content, gave final approval of the version to be published and agreed to be accountable for all aspects of the work.

Correspondence to: Juan Brito, Brito.Juan@mayo.edu 